\title{
The efficacy of multiple versus single hyaluronic acid injections: a systematic review and meta-analysis
}

\author{
Andrew Concoff ${ }^{*}$ (D), Parag Sancheti ${ }^{2}$, Faizan Niazi ${ }^{3}$, Peter Shaw ${ }^{3}$ and Jeffrey Rosen ${ }^{4}$
}

\begin{abstract}
Background: Intra-articular hyaluronic acid (IA-HA) is a common therapy used to treat knee pain and suppress knee inflammation in knee osteoarthritis (OA), typically prescribed in regimens ranging from a single injection to 5 weekly injections given once weekly. We conducted a systematic review to determine the efficacy of IA-HA, with subgroup analyses to explore the differences in knee pain and adverse events (AEs) across different dosing regimens.
\end{abstract}

Methods: We conducted a systematic search of the literature to identify studies evaluating IA-HA for the management of knee OA compared to IA-saline. Primary outcome measure was the mean knee pain score at 13 Weeks (3 months) or 26 weeks (6 months). Secondary outcome was the number of treatment-related AEs and treatment-related serious adverse events (SAEs). We evaluated differences in levels of pain and AEs/SAEs between dosing regimens compared to IA-Saline.

Results: Thirty articles were included. Overall, IA-HA injections were associated with less knee pain compared to IA-Saline injections for all dosing regimens. 2-4 injections of IA-HA vs. IA-Saline produced the largest effect size at both 3-months and 6-months (Standard mean difference $[\mathrm{SMD}]=-0.76$; -0.98 to $-0.53,95 \% \mathrm{Cl}, P<0.00001$, and SMD $=-0.36$; -0.63 to $-0.0995 \% \mathrm{Cl}, P=0.008$, respectively). Additionally, single injection studies yielded a non-significant treatment effect at 3 and 6 months, while $\geq 55$ injections demonstrated a significant improvement in pain only at 6 months. Five or more injections of IA-HA were associated with a higher risk of treatment-related AEs compared to IA-Saline (Risk ratio $[R R]=1.67 ; 1.09$ to $2.5695 \% \mathrm{Cl}, p=0.02$ ), which was a result not seen within the 1 and $2-4$ injection subgroups.

Conclusion: Overall, 2-4 and $\geq 5$ injection regimens provided pain relief over IA-Saline, while single injection did not. Intra-articular injections of HA used in a 2-4 injection treatment regimen provided the greatest benefit when compared to IA-Saline with respect to pain improvement in patients with knee OA, and was generally deemed safe with few to no treatment-related AEs reported across studies. Future research is needed to directly compare these treatment regimens.

Keywords: Intra-articular hyaluronic acid (IA-HA), Osteoarthritis (OA), Knee

\section{Background}

Knee osteoarthritis (OA) is a slowly progressive joint disorder characterized by joint pain, cartilage degeneration, and inflammation that affects approximately 250 million people worldwide [1]. Knee OA leads to negative impacts on socioeconomic factors including impaired work performance and early retirement $[2,3]$.

\footnotetext{
*Correspondence: Andrew.Concoff@stjoe.org

'Departments of Orthopedics and Rheumatology, St. Jude Medical Center,

Fullerton, California, USA

Full list of author information is available at the end of the article
}

Multiple treatment options are available for knee OA, ranging from conservative management to total knee arthroplasty. Common non-operative therapies include non-steroidal anti-inflammatory drugs (NSAIDs), physical therapy, analgesics such as acetaminophen, and intra-articular (IA) therapies such as corticosteroids and hyaluronic acid (HA) [4]. IA-HA products have been used in the United States as a treatment for knee OA since 1997 and are approved by the Food and Drug Administration (FDA) [5]. The putative mechanisms of action through which IA-HA provides therapeutic 
effects include anti-inflammatory effects, chondroprotection, proteoglycan synthesis, and shock absorption properties [6]. IA-HA is frequently used in clinical practice to treat knee pain and suppress knee inflammation.

In 2013, the American Academy of Orthopaedic Surgeons (AAOS) recommended against the use of IAHA for knee OA [7]. However, more recently, certain physician-specialty societies have recommended the use of IA-HA in treating knee OA. The American College of Rheumatology (ACR) recommended IA-HA for knee OA patients who had an inadequate response to initial therapy in a recent position statement [8]. The American Medical Society for Sports Medicine (AMSSM) recommended the use of HA for the appropriate patients with knee OA based on recent evidence in a network metaanalysis [9]. The European Society for Clinical and Economic Aspects of Osteoarthritis (ESCEO) task force issued a consensus statement recommending the use of IA-HA in knee OA patients with mild to moderate disease, and for more severe patients who are not good candidates for total knee replacement surgery or wishing to delay the surgical procedure [10]. The OA Research Society International (OARSI) has an uncertain recommendation for the use of IA-HA in treating knee OA based on the good level of quality of evidence available, suggesting physicians discuss the risk-benefit profile of IA-HA along with individual characteristics, comorbidities and preferences of the patient [11]. Despite the clinical considerations and availability of evidence recommending the use of IA-HA in treating knee OA, the optimal treatment regimen and patient selection criteria have yet to be determined.

IA-HA is commonly prescribed in different injection regimens, which vary from a single injection to one injection a week for 5 weeks. Multiple randomized controlled trials (RCTs) have been conducted evaluating different dosing regimens of IA-HA versus IA-Saline. We conducted a systematic review of the published literature to determine the efficacy of IA-HA vs IA-Saline in patients with knee OA, with subgroup analyses to explore the differences in levels of pain, adverse events (AEs), and serious adverse events (SAEs) across different dosing regimens.

\section{Methods}

\section{Literature search}

A comprehensive literature search for relevant articles was conducted on February 26th, 2016 using a detailed search of the MEDLINE and EMBASE and PubMed databases (Additional file 1). The Preferred Reporting Items for Systematic Reviews and Meta-Analyses (PRISMA) 2009 checklist was applied a posteriori to ensure appropriate reporting of methods and results [12]. The inclusion criteria were: 1 ) blinded randomized controlled trial (RCT) comparing IA-HA with intraarticular saline (IA-Saline) injection; 2) knee pain was a reported outcome; and 3) articles that were published in English. Title screening, abstract screening and full text screening were conducted in duplicate.

\section{Data abstraction}

We abstracted details on the study characteristics, details about the HA product used (manufacturer, production method (Bio-HA [biologically derived/non- animal stabilized] or $\mathrm{AD}$ [avian-derived]) and molecular weight (indicated as high if $\geq 3000 \mathrm{kDa}$, moderate if $<3000$ and $\geq 1500 \mathrm{kDa}$, or low if $<1500 \mathrm{kDa}$ ), the timing of injections, reported pain outcomes, and safety data (the number of treatment-related AEs and treatment-related SAEs). Data extraction was completed by one reviewer, and another reviewer completed a review of the data for accuracy.

\section{Outcome measures}

The primary outcome measure was the mean knee pain score at the reported follow-up nearest to 13 Weeks (3 months) or 26 weeks (6 months). The Western Ontario and McMaster Universities Arthritis Index (WOMAC) Pain scores were extracted whenever reported. If WOMAC pain scores were not reported an a priori hierarchy of outcomes was used to extract the next-most relevant outcome measure. The hierarchy used was taken from a previous metaanalysis, and is as follows: WOMAC Pain, Visual Analog Scale (VAS) Pain with activity/walking, VAS Pain weight bearing, VAS pain at rest, Other Pain outcomes (Knee Injury and Osteoarthritis Outcome Score (KOOS), Musculoskeletal Outcomes Data Evaluation and Management System (MODEMS), Index of Severity for Osteoarthritis for the Knee (ISK) assessment), WOMAC Total Score [13].

We extracted safety data from each trial on total number of participants experiencing treatment-related adverse events (AEs) and total number of treatmentrelated serious adverse events (SAEs). If data for these safety measures was not reported, the corresponding study was not included in the meta-analysis for that specific safety measure. Data from the intent-to-treat population was used whenever possible. Data extraction was completed in duplicate by two reviewers.

\section{Data analysis}

The bias corrected (Hedges) effect size for each trial was calculated using an online Excel calculation tool [14]. If the article did not provide the mean pain score and standard deviation/standard error, then the effect sizes were obtained from recently published systematic reviews and meta-analyses [4, 15]. Standard error was calculated from the confidence intervals (CI) of the effect sizes taken from the meta-analysis using the following equation: 


$$
\text { Standard error }=\frac{\text { High C.I.value-Low C.I.value }}{3.92}
$$

Effect size results and AEs were analyzed using the Cochrane Review Manager 5.3 software [16]. Negative effect size estimates represent benefit of IA-HA, while positive effect size estimate values represent benefit of IA-Saline. Effect size and $\mathrm{AE}$ analyses were separated and pooled based on the number of injections of IA-HA and IA-Saline (single injection, 2-4 injections, or $\geq 5$ injections). Effect size estimates were analyzed using a generic inverse variance statistical method and a random effects analysis model with a $95 \%$ confidence interval for study and total effect size. Effect sizes were reported as a standard mean difference outcome measurement. The number of participants experiencing a treatment-related adverse event and treatment-related SAEs were analyzed under a dichotomous outcome assessment using Mantel-Haenzel statistical method and a fixed effects analysis model with a $95 \%$ confidence interval for study and total effect size. Heterogeneity within the included trials was measured using the $\mathrm{I}^{2}$ statistical measurement. Two reviewers independently graded the methodological quality of each included study using the Cochrane Collaboration's Risk of Bias tool. The Cochrane Risk of Bias tool separates judgments about risk of bias from inadequate reporting of methodology. Post-hoc funnel plot analyses at 3-months and at 6-months were conducted to assess publication bias (Additional file 2: Figure S1 and Additional file 3: Figure S2).

A post-hoc subgroup analysis was conducted comparing the efficacy of IA-HA by total dose administered to determine whether repeated injection or total dose received likely explained the differences observed by number of injections. Studies were separated based on the total dose of IA-HA participants were given (0-60 mg, 61-100 mg, >100 mg).

\section{Sensitivity analysis}

A sensitivity analysis was conducted to determine if single-blinded studies had a significant impact on the total treatment effect of IA-HA on knee-pain vs IASaline. To accomplish this, single-blinded studies were removed from analyses to determine if they had a significant impact on treatment efficacy. Another sensitivity analysis was conducted to determine if removing each study from the 3-month and 6-month treatment effect meta-analyses converted a statistically significant combined difference into a nonsignificant difference.

\section{Results}

\section{Search strategy}

Our literature search identified 2198 articles and 166 of these articles were deemed relevant following the title review (Fig. 1). Of these, 28 articles met the pre-defined inclusion criteria. One study used arthrocentesis as a comparator and was included after careful review [17]. Two additional articles were identified from our review of the reference lists of relevant articles. Therefore, 30 articles were included in our systematic review [17-46].

\section{Study characteristics and demographics}

Most studies were published within the last decade, and were most frequently conducted in Europe (63.3\%), followed by North America (23.3\%), Asia (10.0\%) and Australia (3.3\%) (Table 1). There were 26 double-blinded RCTs (86.7\%) and four single-blinded RCTs (13.3\%) included in this review (Table 2). A total of 5848 patients are included in our analysis. Four studies $(N=1196)$ used single injections of IA-HA, 16 studies $(N=2865)$ used 2-4 injections, and 11 studies $(N=1847)$ evaluated $\geq 5$ injections. One study $(N=63)$ reported administering 1-11 injections of IA-HA and was included in both the $2-4$ injections subgroup and $\geq 5$ injections subgroup [27]. The most common follow-up period was 26 weeks (6 months) (ranging from 4 weeks to 52 weeks).

Low molecular weight HA was used most frequently (47\%), followed by high molecular weight HA (43\%) and moderate weight HA (10\%) (Table 3). The majority of IA-HA products $(63.3 \%)$ were produced via avianderived molecules (ADHA), and through bacterial processes of biological fermentation (Bio-HA) (33.3\%). One study (3.3\%) did not report the IA-HA product used. More than half of the included studies did not report the injection method used (64.5\%); however, the most reported method of injection was either a lateral or medial approach. Injection regimens were fairly consistent amongst treatment groups, reporting similar concentrations of HA preparations (approximately $10 \mathrm{mg} / 2 \mathrm{ml}$ to $30 \mathrm{mg} / 3 \mathrm{ml}$ ) and volume of HA and saline solutions administered ( $2.0 \mathrm{ml}$ to $3.0 \mathrm{ml}$ range).

\section{IA-HA versus IA-saline: Follow-up closest to 3 months (13 weeks)}

Length of follow-up for included studies with nearest to 3-month follow up data ranged from four weeks up to 16 weeks (Fig. 2). Single injection was comprised of only one estimable study [20] (Standard mean difference [SMD] $=-0.03 ;-0.29$ to 0.23$) .2-4$ injections of IA-HA vs. IA-Saline produced the largest effect size of the subgroups (SMD $=-0.76 ;-0.98$ to $-0.53,95 \% \mathrm{CI}, P<$ $0.00001)$. $\geq 5$ injections of IA-HA vs. IA-Saline produced a non-significant effect size estimate of -0.20 $(-0.43$ to $0.03,95 \% \mathrm{CI}, P=0.09)$. Test for subgroup differences were significant $(P<0.00001)$. Heterogeneity was only observed for studies in the 2-4 injections subgroup $\left(\mathrm{I}^{2}=19 \%\right)$. 


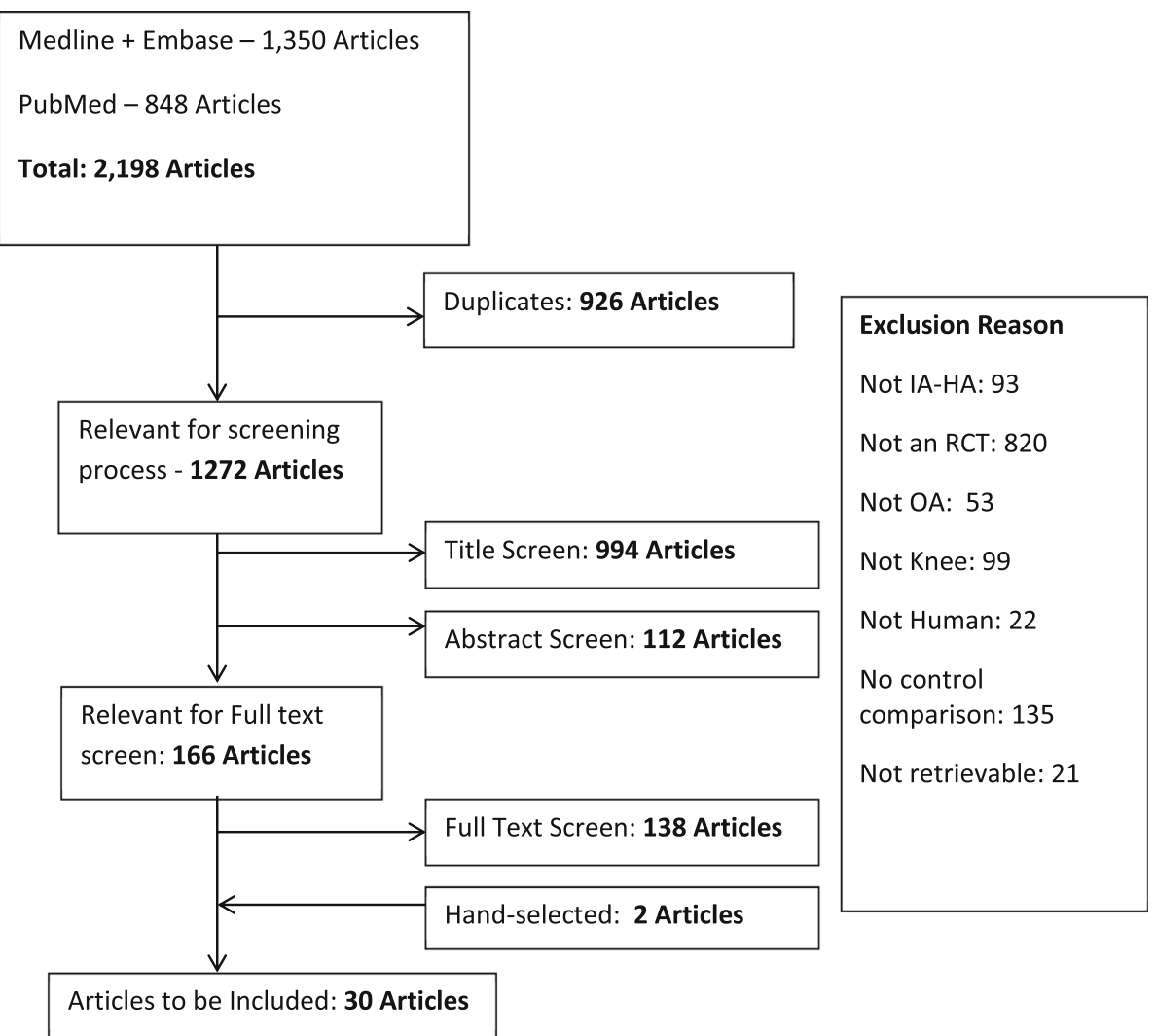

Fig. 1 Screening process. Legend: IA-HA: Intra-articular hyaluronic acid, RCT: Randomized controlled trial, OA: Osteoarthritis

Table 1 Study Location and Year of Publication

\begin{tabular}{ll}
\hline Characteristic & Total $(\%)(N=30)$ \\
\hline Year of Publication & $1(3.3)$ \\
1985-1989 & $4(13.3)$ \\
$1990-1994$ & $3(10.0)$ \\
$1995-1999$ & $6(20.0)$ \\
$2000-2004$ & $8(26.7)$ \\
$2005-2009$ & $7(23.3)$ \\
$2010-2014$ & $1(3.3)$ \\
$2015-2016$ & \\
Study Location & $19(63.3)$ \\
Europe & $7(23.3)$ \\
North America & $3(10.0)$ \\
Asia & $1(3.3)$ \\
Australia & $0(0.0)$ \\
South America & $0(0.0)$ \\
Africa &
\end{tabular}

IA-HA versus IA-saline: Follow-up closest to 6 months (26 weeks)

Length of follow-up for included studies with nearest to 6month follow up data ranged from 18 weeks up to 52 weeks (Fig. 3). Single injection studies yielded a non-significant treatment effect (SMD $=-0.04 ;-0.20$ to $0.13,95 \% \mathrm{CI}, P=$ 0.67). 2-4 injections of IA-HA vs. IA-Saline produced the largest significant effect size $(\mathrm{SMD}=-0.36 ;-0.63$ to -0.09 $95 \% \mathrm{CI}, \mathrm{P}=<0.00001)$. Studies with $\geq 5$ injections of IA-HA vs. IA-Saline produced a significant effect size estimate of -0.18 ( -0.35 to $0.01,95 \% \mathrm{CI}, P=0.04)$. Heterogeneity was observed for studies in the 2-4 injections subgroup $\left(\mathrm{I}^{2}=82 \%\right)$ and $\geq 5$ injections subgroup $\left(\mathrm{I}^{2}=74 \%\right)$.

\section{Efficacy of IA-HA vs IA-saline - Dosage comparison}

No significant subgroup difference were observed when studies were analyzed by total dose of IA-HA administered ( $P=0.90$; Fig. 4). Studies failing to report dosage of IA-HA administered were removed from analysis $[21,24,32,33,35,38,39,46]$.

\section{Treatment-related adverse events and serious adverse events for IA-HA vs. IA-saline}

There were no statistically significant differences in the total number of treatment-related AEs compared to saline 


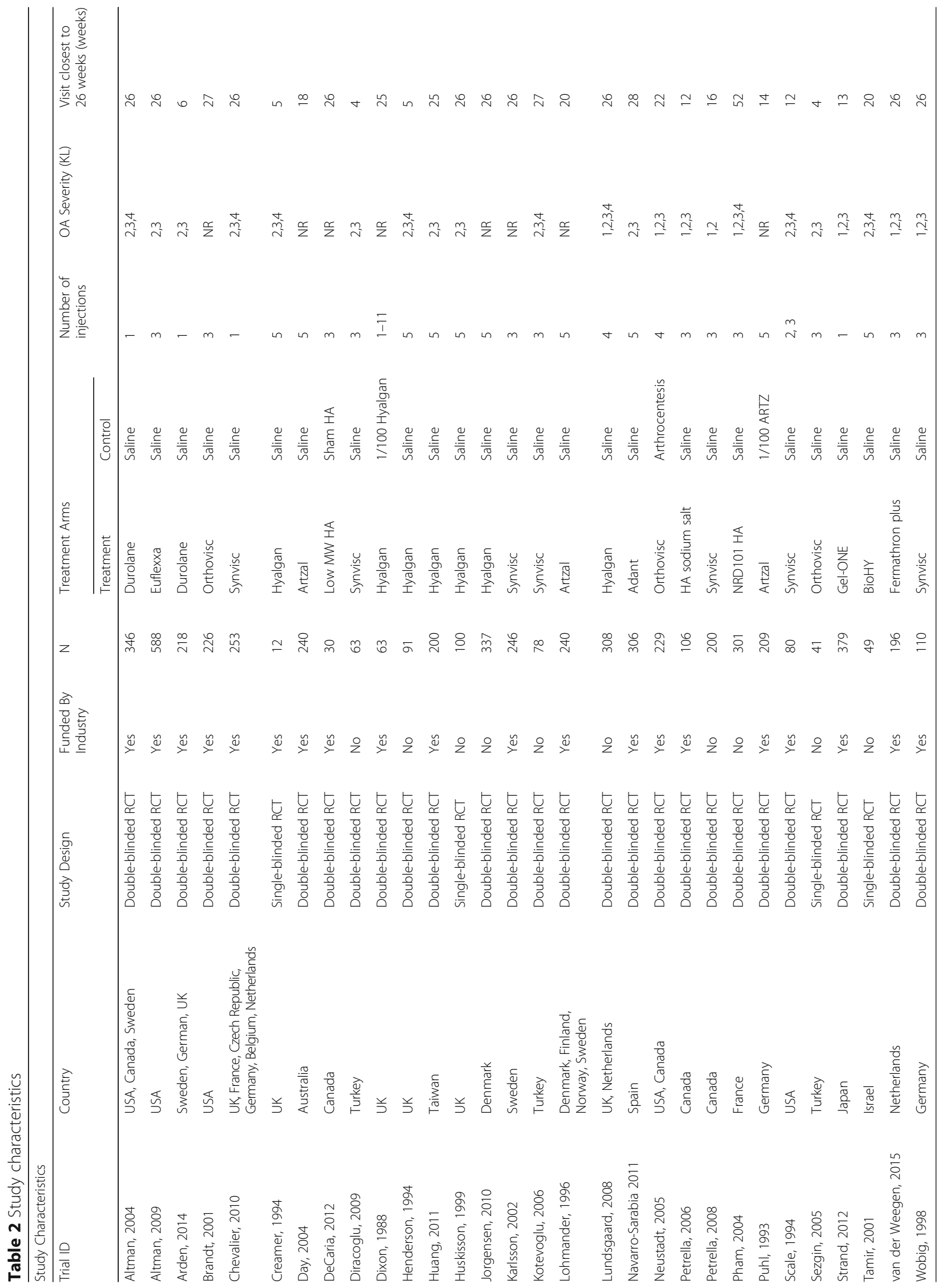




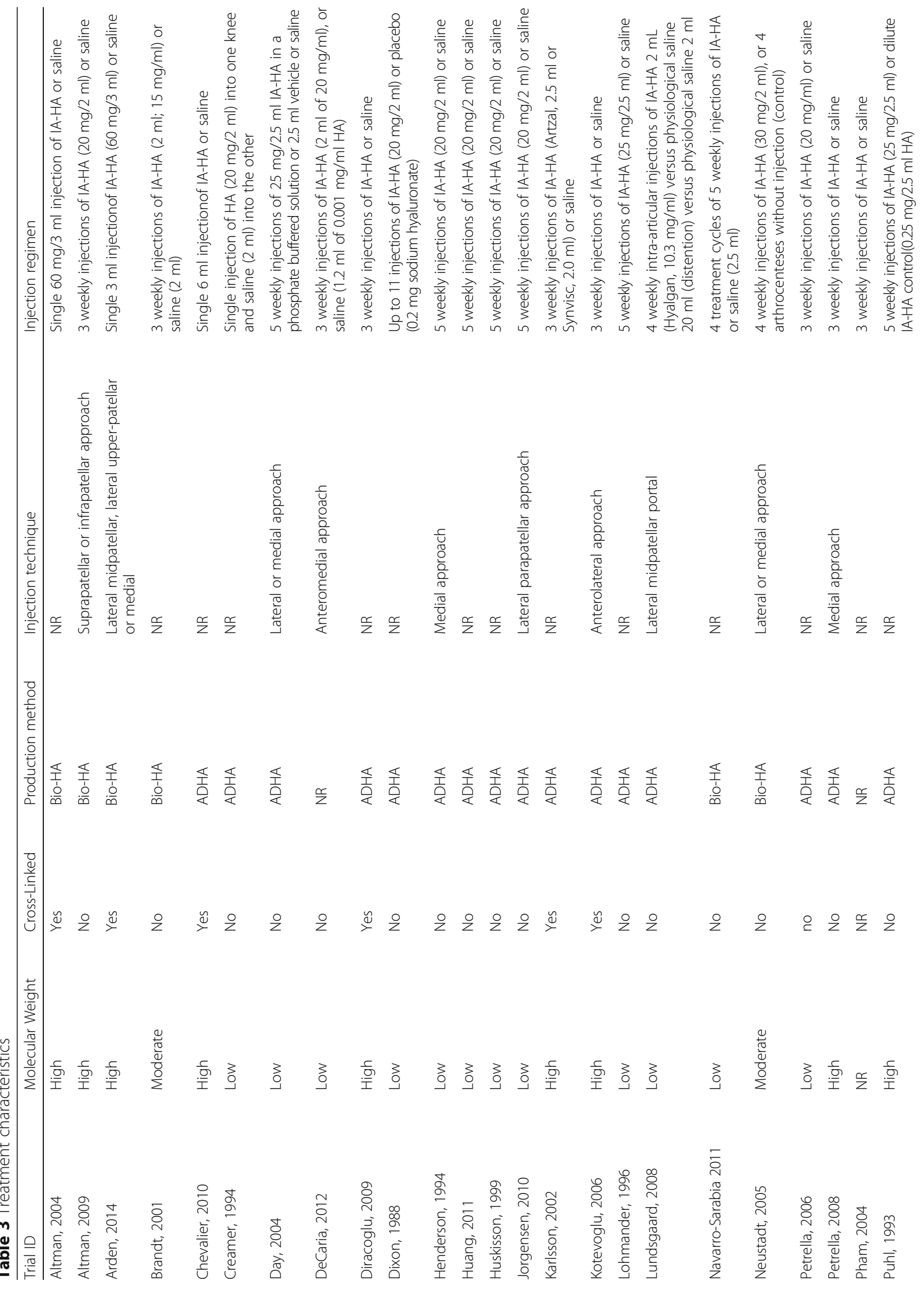




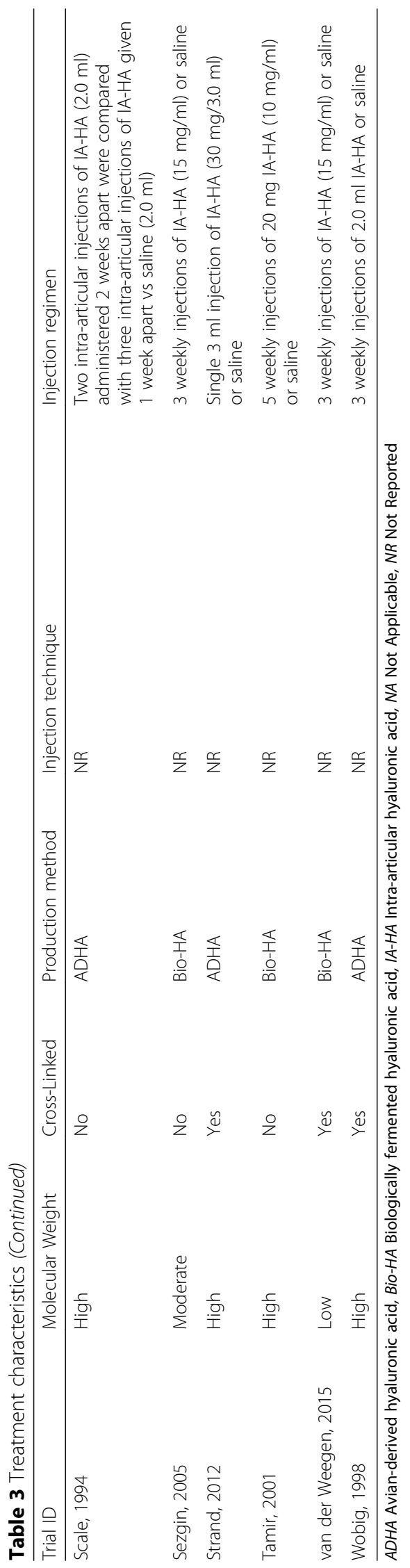




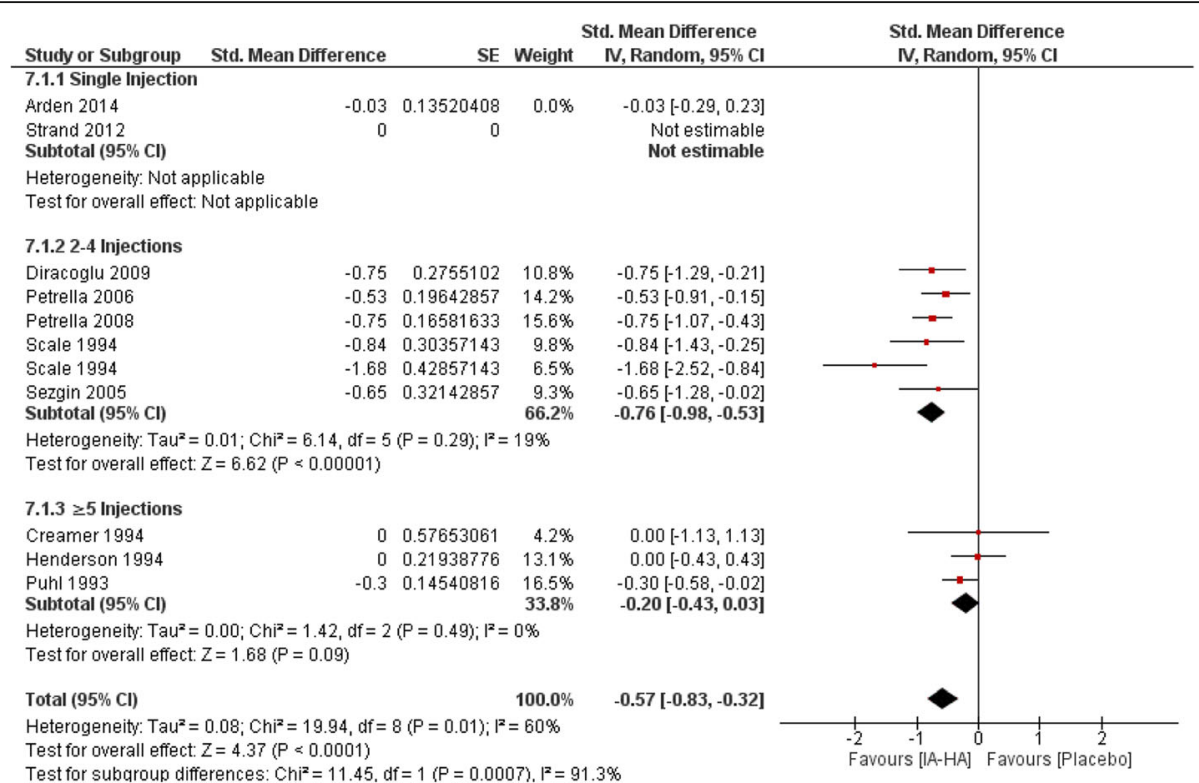

Fig. 2 Efficacy of IA-HA injections closest to 3-months

injection for single injection of IA-HA vs IA-Saline (Risk ratio $[\mathrm{RR}]=1.11 ; 0.93$ to $1.3295 \% \mathrm{CI}, p=0.26$ ) or 2-4 injections of IA-HA vs IA-Saline $(\mathrm{RR}=0.98$; 0.87 to $1.0995 \% \mathrm{CI}, p=0.67$; Fig. 5). Studies with $\geq 5$ injections of IA-HA had statistically more treatment- related AEs compared to IA-Saline $(\mathrm{RR}=1.70 ; 1.12$ to 2.59 95\% CI, $p=0.01$ ). Significant subgroup differences were observed between number of injections and treatment-related AEs $(P=0.03)$, but not for treatment-related SAEs (Fig. 6).

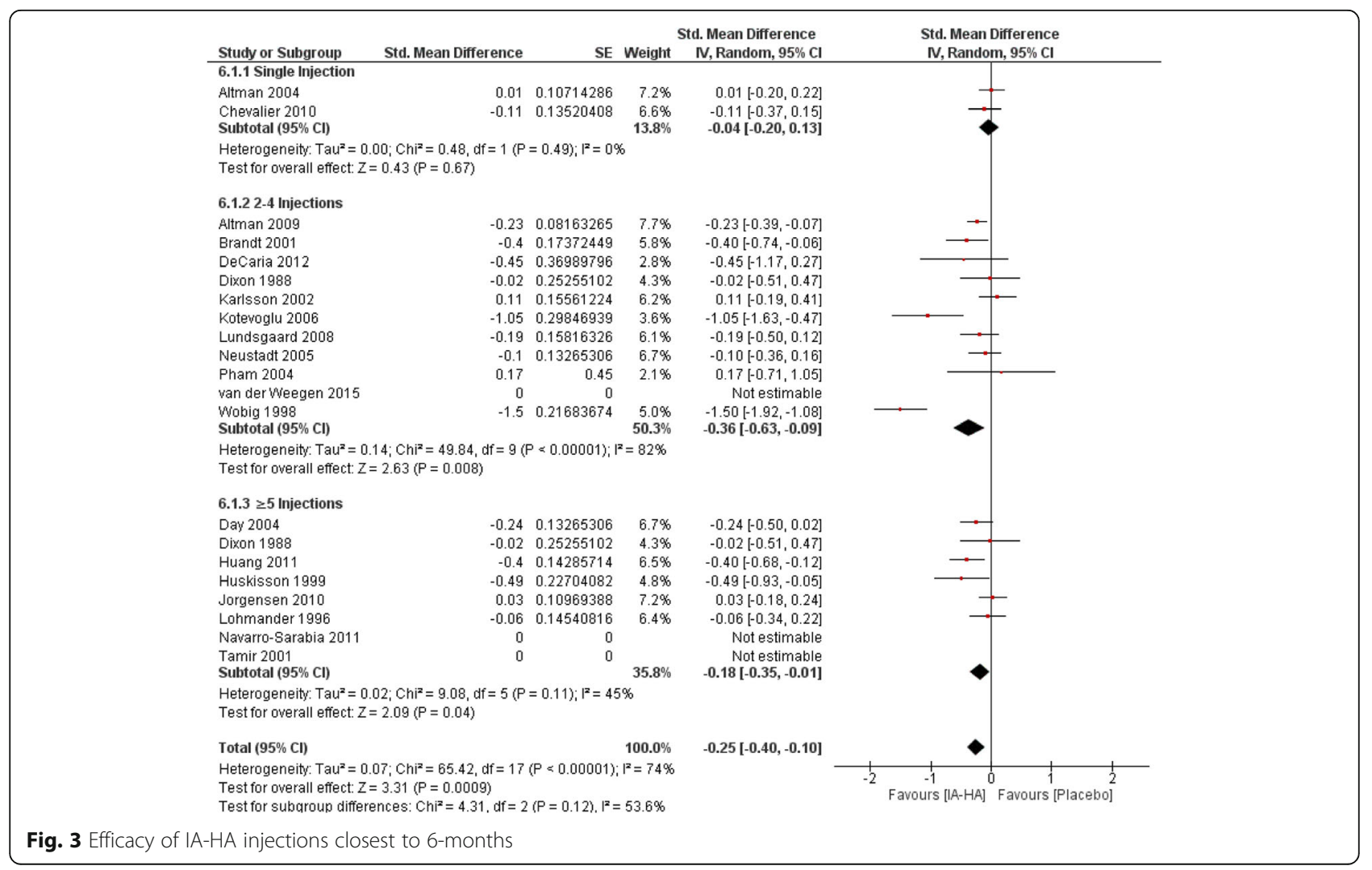




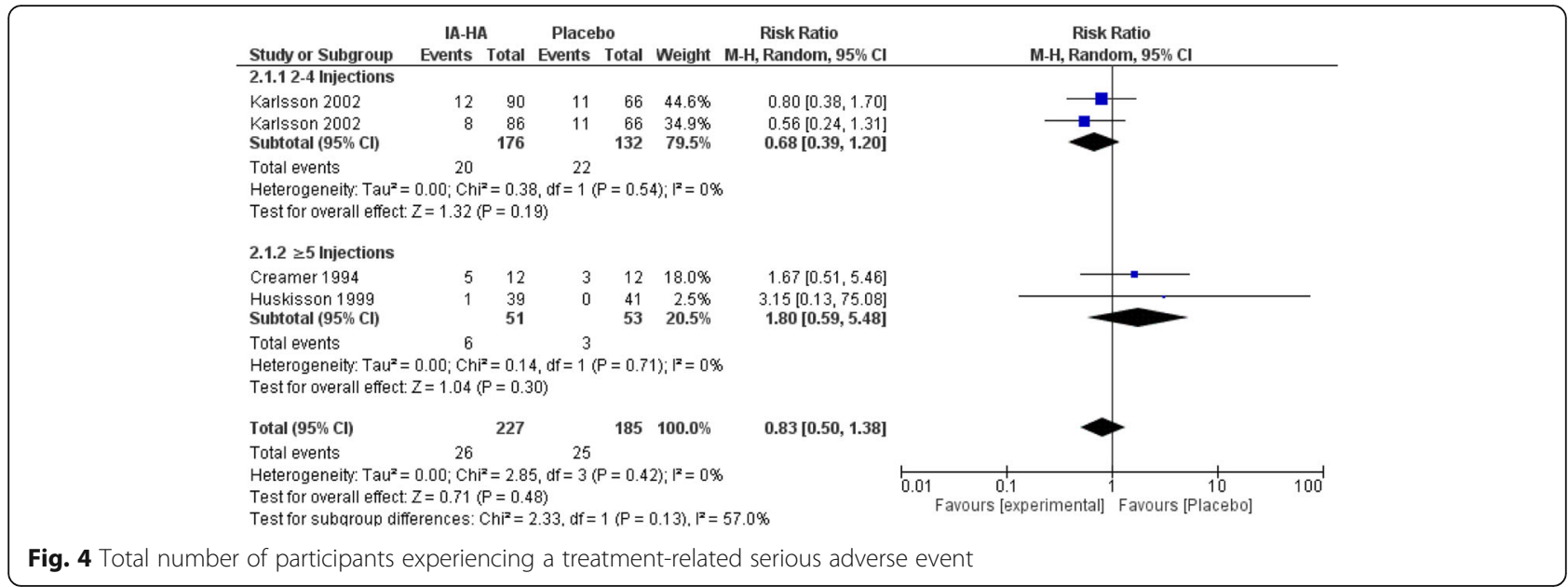

\section{Sensitivity analysis}

Four single-blinded studies were removed from the sensitivity analysis [23, 30, 42, 44]. The pooled effect size remained statistically significant with little change in total effect size when these single-blind studies were removed from the analysis $(\mathrm{SMD}=$ $-0.19[-0.25,-0.13], P<0.001)$. In studies with a follow-up closest to 6 months (26 weeks), removing one study in the $\geq 5$ injections subgroup $[24,29,30]$ changed the subgroup results from significant to non-significant.

\section{Risk of bias}

Included studies demonstrated minimal bias with respect to categories of selection bias, detection bias, performance bias, attrition bias and reporting bias (Fig. 7). Few studies failed to report methods of randomization and methods of blinding.

\section{Discussion}

Overall, treatment with IA-HA was observed to be more effective in treating patients with OA knee pain compared to IA-Saline, with 2-4 injections demonstrating

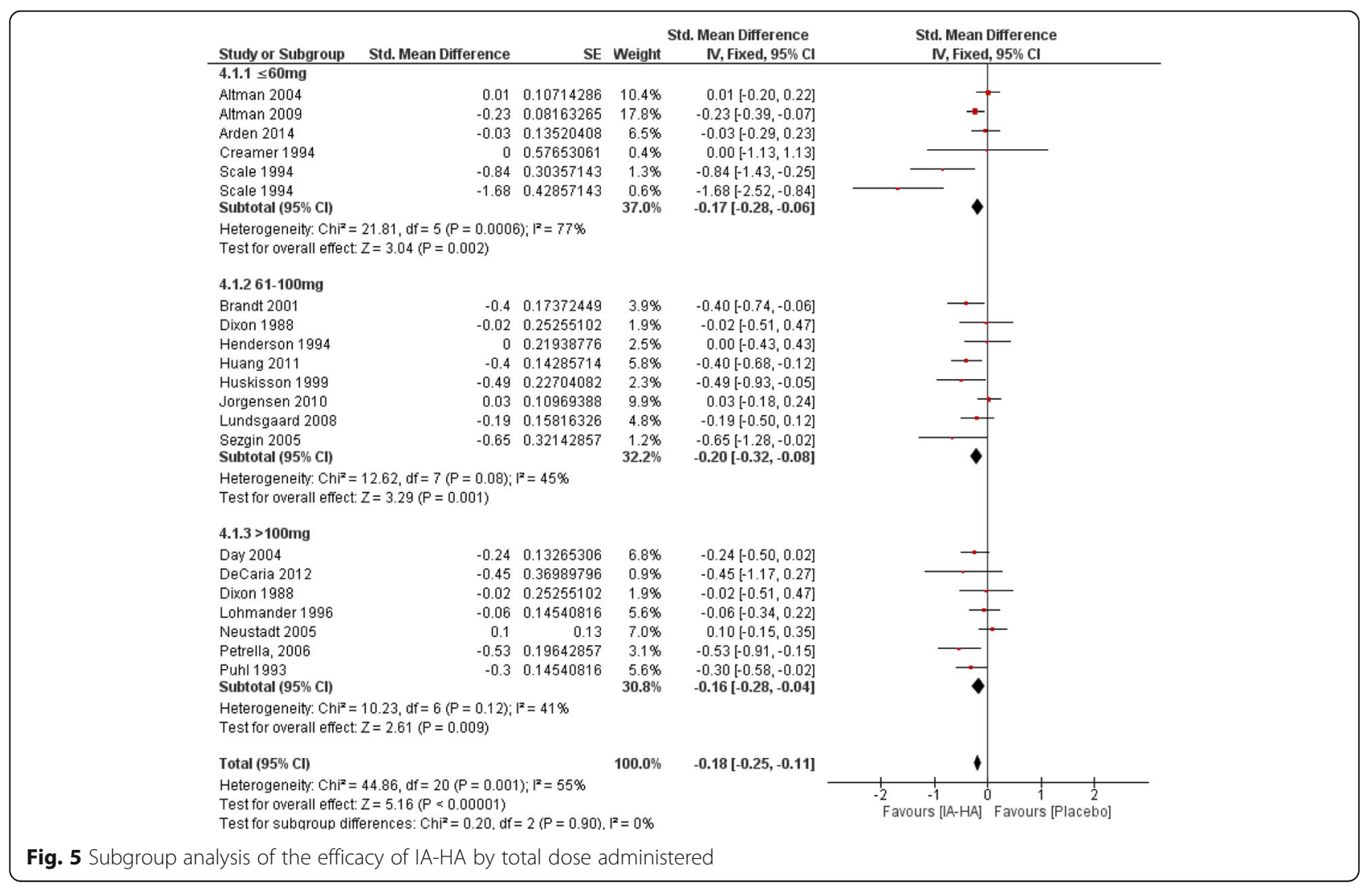




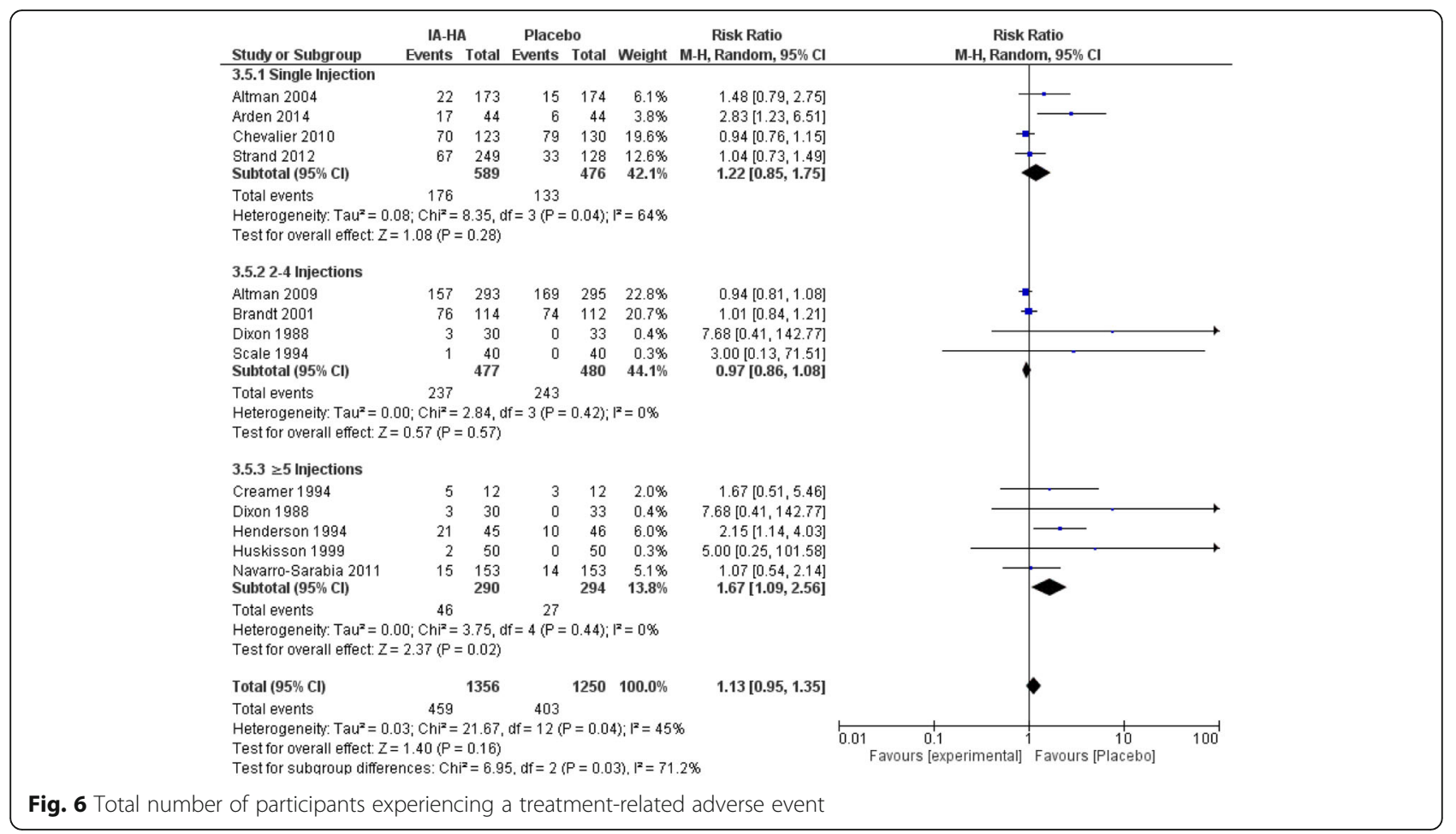

the largest treatment effect at both 3-month and 6month follow-ups. Single injections of IA-HA were not significantly more effective than saline at 3-month or 6month follow ups; however there were only four published RCTs comparing single injections with one trial not reporting the effect size. The $\geq 5$ injections subgroup demonstrated significant improvement in pain at 6 months only. These results indicate that only the 2-4 injection regimen group provided consistently significant pain relief at both 3 and 6 months follow-up, when compared to IA-Saline.

Our results showed greatest improvement at 3 months, while improvement was seen to a lesser extent at 6 months following IA-HA. This is similar to the therapeutic trajectory of IA-HA vs IA-Saline conducted by Bannuru and colleagues [47]. Effect sizes favored IA-HA by week $4((0.31 ; 95 \% \mathrm{CI} 0.17,0.45)$ and peak at week 8 $(0.46 ; 0.28,0.65)$, suggesting that the optimal improvement is seen around 2 months after IA-HA injection. A network meta-analysis conducted by Bannuru et al [4] found similar results when comparing IA-HA to saline controls; IA-HA was favoured over IA-Saline control $(\mathrm{SMD}=0.429$; C.I. 0.261 to $0.598, p=0.000)$ on knee pain at 4 to 13 weeks of treatment. Another network meta-analysis demonstrated similar conclusions, as IAHA was seen to have a significant effect on pain when compared to IA-Saline [48]. Our analysis has demonstrated that not only does the overall pooled estimate for HA as a class demonstrate a benefit for pain relief, there seem to be differences in effect as a result of the injection regimen provided. Previous analyses have suggested that in addition to HA class benefits in pain relief,

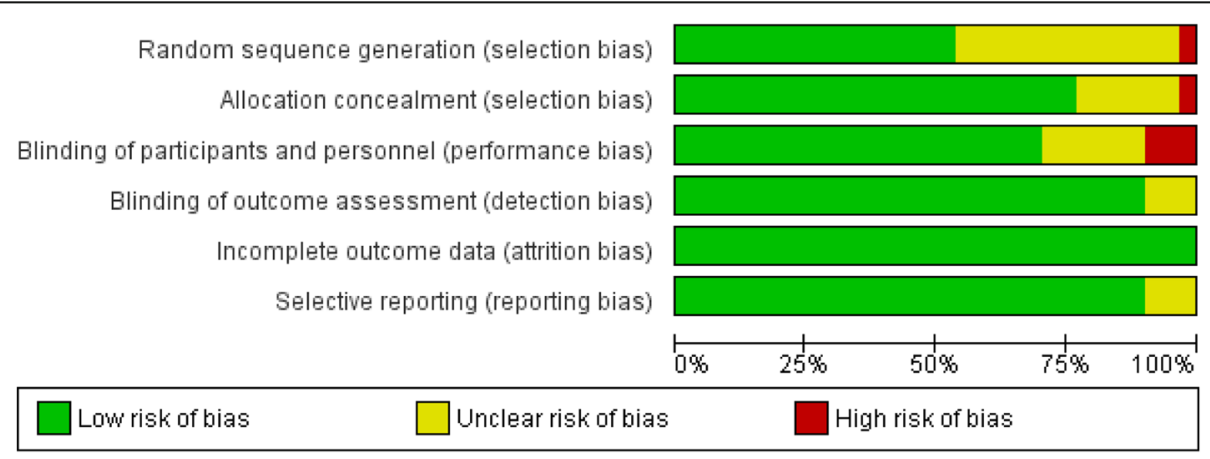

Fig. 7 Risk of bias summary 
molecular weight may also be a product characteristic that affects the potential outcomes of patients [13, 15].

The results of this meta-analysis of knee pain efficacy for 1 injection, 2-4 injections and $\geq 5$ injections of IAHA concur with a recently published RCT [49] in suggesting that the dosing regimen of IA-HA should be considered for development of future guidelines in treating symptomatic knee OA. Zoboli et al. published a head-to-head comparison, RCT assessing whether a single $6 \mathrm{ml}$ application of HA has the same effectiveness as the three-weekly $2 \mathrm{ml}$ dose [49]. Although this study showed no significant differences in efficacy between one single injection and three weekly injections of the same dose of IA-HA administered, the 3 weekly injections regimen showed statistically significant improvement from baseline pain (WOMAC pain and VAS) whereas the single injection regimen did not. Although there were subgroup differences between the numbers of injections administered within this meta-analysis, there was no subgroup difference in efficacy observed when comparing total dose of IA-HA administered to participants vs IA-Saline.

Recent evidence suggests products with an average molecular weight $\geq 3000 \mathrm{kDa}$ provide favorable efficacy results when compared with products of an average molecular weight $<3000 \mathrm{kDa}$, and significantly fewer discontinuations compared with products with a molecular weight $<1500 \mathrm{kDa}^{13}$. In this meta-analysis, we identified that the number of injections may also play a significant role in efficacy outcomes. Although the 2-4 injection subgroup included products within a wide range of MW's the effect size was still significant compared to the single injection subgroup which consisted of only cross-linked high molecular weight (HMW) products. It has been suggested oxygen-derived free radicals act as a mediator in the inflammatory response, and that these radical species are responsible for increased HA degradation [50]. HMW products may achieve better efficacy due to an increased residency time of within the synovial fluid, producing a prolonged anti-inflammatory response within the joint, blocking inflammatory receptors, and a longer lasting chondroprotective effect (inhibition of metalloproteases, nitric oxide, and stimulation of proteoglycan/glycosaminoglycan synthesis) [51-53]. Therefore, repeated exposures of HA may perpetuate improvement in the synovial fluid environment allowing subsequent IA-HA shots to provided extended effects. The results of this study help demonstrate that, while molecular weight is an important factor in the efficacy of HA products, the number of injections provided also plays a major role in optimizing the efficacy seen within knee OA patients. Thus is particularly clear given that, although all single injection HA products were of a high MW, they did not demonstrate a reduction in pain comparable to the 2-4 injections subgroup, which included several studies of LMW HA products. This study demonstrates that receiving the typical 3 injection regimen of a HA may be more effective than a single injection high molecular weight product. Future aims should investigate the mechanism of actions of high molecular weight HA in multiple injections compared to a single injection.

Significantly more treatment-related AEs were observed in participants receiving $\geq 5$ injections vs IASaline; a result not seen in the single and 2-4 injection categories. Although this comparison to IA-Saline was significant, the subgroup analysis comparing the different injection regimens did not show a significant difference in treatment-related AEs between numbers of injection subgroups. Moreover, four of the five studies that reported treatment-related AEs reported a difference of only 1-3 events between treatment groups, and this analysis was largely subjugated by a single study conducted by Henderson et al. ${ }^{256}$ that reported a difference of 11 events, with the IA-HA treatment group experiencing more AEs. This meta-analysis observed very few serious treatment-related AEs, with little to no events reported for all injection regimens. Our findings are similar to the meta-analysis conducted by Miller and Block [54] that focused on the safety and efficacy of US approved IA-HA products in saline-controlled trials, where no SAEs were determined related to injection of HA or saline.

This review has strength in its methodological approach in systematically identifying available salinecontrolled RCTs from online databases. The use of a thorough and systematic approach to article selection and data abstraction provides further strength to this report. A search of the grey literature or unpublished literature was not conducted; however, authors scanned references in articles that met the inclusion criteria for literature not captured in the search. Limitations of this review include that some studies of IA-HA treatment were excluded from the analysis due to not reporting efficacy measurements for knee pain. Another limitation is the lack of a direct comparison between the numbers of injections received, as the literature is not robust enough to permit such analysis. Additionally, the majority of these RCTs were industry funded with a moderate to high risk of bias. Further, the inconsistent reporting of pain scores, along with the variable length of follow-up time between studies provides another limitation with respect to our pooled results. Heterogeneity was also seen within some subgroups, which is an additional limitation to this study. Finally, no assessment or consideration of the effect of the placebo effect in relation to the number of injections was considered within these analyses. 
This review has provided a detailed evaluation of differences between injection regimens of IA-HA for knee pain in OA. Future studies should directly compare different injection regimens of IA-HA in head-to-head RCTs. Moreover, future studies should review alternative outcome measurements such as function, stiffness, and withdrawal rates due to the different number of IA-HA injections vs saline. Further studies should also aim to further compare the different HA products and review intrinsic efficacy and safety profiles of different products based on the number of injections and their molecular weight, structure, and production method. The identification of product-specific results would also allow for greater specificity in drafting clinical guidelines for use of $\mathrm{HA}$ in knee OA. Injection accuracy is also a factor that may contribute to the overall efficacy and safety profile of IA-HA treatment [55], which may warrant future investigation of how accuracy improvement using ultrasound-guided injection techniques may affect clinical outcomes in trials. Additionally, the use of pre-set criteria for response, such as the OMERACT-OARSI responder criteria, is an emerging outcome measurement tool that may provide a more appropriate assessment of individual patient outcomes rather than group mean responses [56]. However, the lack of consistent reporting of this outcome within the current literature precluded the ability to conduct any analysis of OMERACT-OARSI responders between injection regimens [56]. Future studies should consider analyzing measures of individual response according to present criteria for response when designing clinical trials in knee osteoarthritis, including those addressing the impact of IA-HA.

Other factors in addition to injection regimen may contribute to the efficacy and safety of IA-HA products, such as molecular weight and production process. Altman and colleagues [6] concluded that in the available literature, IA-HA products with a molecular weight $\geq 3000 \mathrm{kDa}$ and those derived from biological fermentation relate to superior efficacy and safety [6]. The low molecular weight (LMW) IA-HA pooled effect size did not meet the minimum clinically important difference (MCID) threshold, demonstrating an insignificant clinical effect for pain relief. Due to the multiple variables that may contribute to the efficacy of IA-HA products, additional investigations comparing the different types of HA products are required to fully understand the efficacy differences of IA-HA products in knee OA. Guideline development groups and clinicians should consider the injection regimen for various types of IA-HA treatments in decision-making processes regarding the appropriate use of IA-HA treatment for knee OA.

\section{Conclusion}

Overall, $2-4$ and $\geq 5$ injection regimens provided pain relief over IA-Saline. Intra-articular injections of HA used in a 2-4 injection treatment regimen provided the greatest benefit when compared to IA-Saline with respect to pain improvement in patients with knee OA, and were generally deemed safe with few to no treatment-related AEs reported across studies. Future research is needed to directly compare these treatment regimens, as well as further investigate the effects of other variables, such as product molecular weight, in the comparison of IA-HA injection treatment regimens.

\section{Additional files}

Additional file 1: Literature Search Strategy. (DOCX 15 kb)

Additional file 2: Figure S1. Funnel plot analysis of studies investigating efficacy of IA-HA injections closest to 3-months. (DOCX 18 kb)

Additional file 3: Figure S2. Funnel plot analysis of studies investigating efficacy of IA-HA injections closest to 6-months. (DOCX 19 kb)

\section{Abbreviations}

AAOS: American Academy of Orthopaedic Surgeons; ACR: American College of Rheumatology; AD: Avian-derived; AE: Adverse Events; AMSSM: American Medical Society for Sports Medicine; Cl: Confidence interval; ESCEO: European Society for Clinical and Economic Aspects of Osteoarthritis; FDA: Food and Drug Administration; HWM: High molecular weight; IA-HA: Intra-articular hyaluronic acid; ISK: Index of Severity for Osteoarthritis for the Knee; KOOS: Knee Injury and Osteoarthritis Outcome Score; LMW: Low molecular weight; MCID: Minimum clinically important difference; MODEMS: Musculoskeletal Outcomes Data Evaluation and Management System; MW: Molecular weight; NSAID: Nonsteroidal anti-inflammatory drug; OA: Osteoarthritis; OARSI: Osteoarthritis Research Society International; OMERACT: Outcome measures in rheumatology; PRISMA: Preferred Reporting Items for Systematic Reviews and Meta-Analyses; RCT: Randomized controlled trial; RR: Risk ratio; SAE: Serious adverse events; SMD: Standard mean difference; US: United States; VAS: Visual analog score; WOMAC: Western Ontario and McMaster Universities Arthritis Index

\section{Acknowledgements}

We would like to thank Mark Gichuru and Mark Phillips of Global Research Solutions Inc. for their medical writing assistance throughout this project.

\section{Funding}

This study was funded by Ferring Pharmaceuticals.

\section{Availability of data and materials} Not applicable

\section{Authors' contributions \\ All authors contributed to the systematic review methodology and the drafting of the manuscript. AC, PS, FN, PS, and JR assisted in revising, editing and approving the manuscript. Corresponding author is AC.}

Ethics approval and consent to participate Not applicable

Consent for publication

Not applicable

\section{Competing interests}

Andrew Concoff: Honorarium received for Ferring Pharmaceuticals advisory board. Parag Sancheti: None.

Faizan Niazi: Paid employee of Ferring Pharmaceuticals.

Peter Shaw: Paid employee of Ferring Pharmaceuticals. 
Jeffrey Rosen: Honorarium received for Ferring Pharmaceuticals, Flexion, and Novartis advisory boards. Honorarium received for OrthoGenrx sales force lecture.

\section{Publisher's Note}

Springer Nature remains neutral with regard to jurisdictional claims in published maps and institutional affiliations.

\section{Author details}

'Departments of Orthopedics and Rheumatology, St. Jude Medical Center, Fullerton, California, USA. ${ }^{2}$ Sancheti Institute for Orthopaedics and Rehabilitation, Maharashtra, India. ${ }^{3}$ Ferring Pharmaceuticals Inc., Parsippany, NJ, USA. ${ }^{4}$ Department of Orthopaedics \& Rehabilitation, New York Presbyterian Queens; Department of Clinical Orthopaedic Surgery, Weill Medical College of Cornell University, New York, NY, USA

Received: 21 June 2017 Accepted: 8 December 2017

Published online: 21 December 2017

\section{References}

1. Vos T, Flaxman AD, Naghavi M, et al. Years lived with disability (YLDs) for 1160 sequelae of 289 diseases and injuries: a systematic analysis for the global burden of disease study 2010. Lancet. 2012;380(9859):2163-96.

2. Cheng OT, Souzdalnitski D, Vrooman B, Cheng J. Evidence-based knee injections for the Management of Arthritis. Pain Med (United States). 2012; 13(6):740-53.

3. Kosorok MR, Omenn GS, Diehr P, Koepsell TD, Patrick DL. Restricted activity days among older adults. Am J Public Health. 1992;82(9):1263-7.

4. Bannuru RR, Schmid CH, Kent DM, Vaysbrot EE, Wong JB, McAlindon TE. Comparative effectiveness of pharmacologic interventions for knee osteoarthritis: a systematic review and network meta-analysis. Ann Intern Med. 2015;162(1): 46-54. https://doi.org/10.7326/M14-1231.

5. Strand V, Conaghan PG, Lohmander LS, et al. An integrated analysis of five double-blind, randomized controlled trials evaluating the safety and efficacy of a hyaluronan product for intra-articular injection in osteoarthritis of the knee. Osteoarthr Cartil. 2006;14(9):859-66.

6. Altman RD, Manjoo A, Fierlinger A, Niazi F, Nicholls M. The mechanism of action for hyaluronic acid treatment in the osteoarthritic knee: a systematic review. BMC Musculoskelet Disord. 2015;16:321.

7. American Academy of Orthopaedic Surgeons Academy, AAOS. Treatment of Osteoarthritis of the Knee Evidence-Based Guideline $2^{\text {nd }}$ Edition. Adopted by the American Academy of Orthopaedic Surgeions Board of Directors. Am Acad Orthop Surg Board Dir. 2013:973.

8. American College of Rheumatology. Position Statement. Subject: IntraArticular Hyaluronic Acid Injection in Osteoarthritis of the Knee. Available at https://www.rheumatology.org/Portals/0/Files/Viscosupplementation.pdf Accessed September 26, 2017.

9. Trojian T, Concoff A, Joy S, Hatzenbuehler J, Saulsberry W, Coleman CAMSSM Scientific statement concerning viscosupplementation injections for knee osteoarthritis: importance for individual patient outcomes. Br J Sports Med. 2016;50(2):84-92

10. Bruyère $\mathrm{O}$, Cooper $\mathrm{C}$, Pelletier J, et al. A consensus statement on the European Society for Clinical and Economic Aspects of osteoporosis and osteoarthritis (ESCEO) algorithm for the management of knee osteoarthritis-from evidence-based medicine to the real-life setting. Semin Arthritis Rheum. 2016:45(4):S3-S11.

11. McAlindon T, Bannuru R, Sullivan M, et al. OARSI guidelines for the nonsurgical management of knee osteoarthritis. Osteoarthr Cartil. 2014;22(3): 363-88

12. Moher D, Liberati A, Tetzlaff J, Altman D. Preferred reporting items for systematic reviews and meta-analyses: the PRISMA statement. PLoS Med. 2009;6(7):e1000097.

13. Altman RD, Bedi A, Karlsson J, Sancheti P, Schemitsch E. Product Differences in Intra-articular Hyaluronic Acids for Osteoarthritis of the Knee. Am J Sports Med. Nov 172015.

14. Coe R. Effect Size Calculator. http://www.cem.org/effect-size-calculator. Published 2016

15. Rutjes AWS, Juni $P$, da Costa BR, Trelle S, Nuesch E, Reichenbach S. Viscosupplementation for osteoarthritis of the knee: a systematic review and meta-analysis. Ann Intern Med. 2012;157(3):180-91.

16. Review Manager (RevMan) Version 5.3. Copenhagen: The Nordic Cochrane Centre, The Cochrane Collaboration, 2014
17. Lohmander L, Dalén N, Englund G, et al. Intra-articular hyaluronan injections in the treatment of osteoarthritis of the knee: a randomised, double blind, placebo controlled multicentre trial. Ann Rheum Dis. 1996;55:424-31.

18. Altman RD, Akermark C, Beaulieu AD, et al. Efficacy and safety of a single intra-articular injection of non-animal stabilized hyaluronic acid (NASHA) in patients with osteoarthritis of the knee. Osteoarthr Cartil. 2004:12(8):642-9.

19. Altman RD, Rosen JE, Bloch DA, Hatoum HT, Korner PA. Double-blind, randomized, saline-controlled study of the efficacy and safety of EUFLEXXA for treatment of painful osteoarthritis of the knee, with an open-label safety extension (the FLEXX trial). Semin Arthritis Rheum. 2009;39(1):1-9.

20. Arden NK, Akermark C, Andersson M, Todman MG, Altman RDA. Randomized saline-controlled trial of NASHA hyaluronic acid for knee osteoarthritis. Curr Med Res Opin. 2014:30(2):279-86.

21. Brandt KD, Block JA, Michalski JP, Moreland LW, Caldwell JR, Lavin PT. Efficacy and safety of intraarticular sodium hyaluronate in knee osteoarthritis. ORTHOVISC study. Group. 2001

22. Chevalier $X$, Jerosch J, Goupille $P$, et al. Single, intra-articular treatment with $6 \mathrm{ml}$ hylan G-F 20 in patients with symptomatic primary osteoarthritis of the knee: a randomised, multicentre, double-blind, placebo controlled trial. Ann Rheum Dis. 2010;69(1):113-9.

23. Creamer P, Sharif M, George E, et al. Intra-articular hyaluronic acid in osteoarthritis of the knee: an investigation into mechanisms of action. Osteoarthr Cartil. 1994;2(2):133-40.

24. Day R, Brooks P, Conaghan PG, Petersen MA. Double blind, randomized, multicenter, parallel group study of the effectiveness and tolerance of intraarticular Hyaluronan in osteoarthritis of the knee. J Rheumatol. 2004; 31(4):775-82.

25. DeCaria JE, Montero-Odasso M, Wolfe D, Chesworth BM, Petrella RJ. The effect of intra-articular hyaluronic acid treatment on gait velocity in older knee osteoarthritis patients: a randomized, controlled study. Arch Gerontol Geriatr. 2012;55(2):310-5

26. Diracoglu D, Vural M, Baskent A, Dikici F, Aksoy C. The effect of viscosupplementation on neuromuscular control of the knee in patients with osteoarthritis. J Back Musculoskelet Rehabil. 2009;22(1):1-9.

27. Dixon A, Jacoby R, Berry H, Hamilton E. Clinical trial of intra-articular injection of sodium hyaluronate in patients with osteoarthritis of the knee. Curr Med Res Opin. 1988;11(4):205-13.

28. Henderson EB, Smith EC, Pegley F, Blake DR. Intra-articular injections of 750 $\mathrm{KD}$ hyaluronan in the treatment of osteoarthritis: a randomised single centre double-blind placebo-controlled trial of 91 patients demonstrating lack of efficacy. Ann Rheum Dis. 1994;53(8):529-34.

29. Huang TL, Chang CC, Lee CH, Chen SC, Lai CH, Tsai CL. Intra-articular injections of sodium hyaluronate (Hyalgan $\left.{ }^{\oplus}\right)$ in osteoarthritis of the knee. A randomized, controlled, double-blind, multicenter trial in the Asian population. BMC Musculoskelet Disord. 2011:12:221.

30. Huskisson EC, Donnelly S. Hyaluronic acid in the treatment of osteoarthritis of the knee. Rheumatology (Oxford). 1999;38(7):602-7.

31. Jorgensen A, Stengaard-Pedersen K, Simonsen O, et al. Intra-articular hyaluronan is without clinical effect in knee osteoarthritis: a multicentre, randomised, placebo-controlled, double-blind study of 337 patients followed for 1 year. Ann Rheum Dis. 2010;69(6):1097-102.

32. Karlsson J, Sjögren LS, Lohmander LS. Comparison of two hyaluronan drugs and placebo in patients with knee osteoarthritis. A controlled, randomized, double-blind, parallel-design multicentre study. Rheumatology (Oxford). 2002:41(11):1240-8.

33. Kotevoglu N, lyibozkurt PC, Hiz O, Toktas H, Kuran BA. Prospective randomised controlled clinical trial comparing the efficacy of different molecular weight hyaluronan solutions in the treatment of knee osteoarthritis. Rheumatol Int. 2006;26(4):325-30

34. Lundsgaard C, Dufour N, Fallentin E, Winkel P, Gluud C. Intra-articular sodium hyaluronate $2 \mathrm{~mL}$ versus physiological saline $20 \mathrm{~mL}$ versus physiological saline $2 \mathrm{~mL}$ for painful knee osteoarthritis: a randomized clinical trial. Scand J Rheumatol. 2008;37(2):142-50

35. Navarro-Sarabia F, Coronel P, Collantes E, et al. A 40-month multicentre, randomised placebo-controlled study to assess the efficacy and carry-over effect of repeated intra-articular injections of hyaluronic acid in knee osteoarthritis: the AMELIA project. Ann Rheum Dis. 2011;70(11):1957-62.

36. Neustadt D, Caldwell J, Bell M, Wade J, Gimbel J. Clinical effects of intraarticular injection of high molecular weight hyaluronan (Orthovisc) in osteoarthritis of the knee: a randomized, controlled, multicenter trial. J Rheumatol, 2005:32(10): 1928-36. 
37. Petrella RJ, Petrella MA. Prospective, randomized, double-blind, placebo controlled study to evaluate the efficacy of intraarticular hyaluronic acid for osteoarthritis of the knee. J Rheumatol. 2006;33(5):951-6.

38. Petrella RJ, Cogliano A, Decaria J. Combining two hyaluronic acids in osteoarthritis of the knee: a randomized, double-blind, placebo-controlled trial. Clin Rheumatol. 2008;27(8):975-81.

39. Pham T, Le Henanff A, Ravaud P, Dieppe P, Paolozzi L, Dougados M. Evaluation of the symptomatic and structural efficacy of a new hyaluronic acid compound, NRD101, in comparison with diacerein and placebo in a 1 year randomised controlled study in symptomatic knee osteoarthritis. Ann Rheum Dis. 2004;63(12):1611-7.

40. Puhl W, Bernau A, Greiling $H$, et al. Intra-articular sodium hyaluronate in osteoarthritis of the knee: a multicenter, double-blind study. Osteoarthr Cartil Osteoarthr Res Soc. 1993;1:233-41.

41. Scale D, Wobig M, Wolpert W. Viscosupplementation of osteoarthritic knees with hylan: a treatment schedule study. Curr Ther Res. 1994;55(3):220-32.

42. Sezgin $M$, Demirel A, Karaca C, et al. Does hyaluronan affect inflammatory cytokines in knee osteoarthritis? Rheumatol Int. 2005;25(4):264-9.

43. Strand V, Baraf HSB, Lavin PT, Lim S, Hosokawa HA. Multicenter, randomized controlled trial comparing a single intra-articular injection of Gel-200, a new cross-linked formulation of hyaluronic acid, to phosphate buffered saline for treatment of osteoarthritis of the knee. Osteoarthr Cartil. 2012;20(5):350-6.

44. Tamir E, Robinson D, Koren R, Agar G, Halperin N. Intra-articular hyaluronan injections for the treatment of osteoarthritis of the knee: a randomized, double blind, placebo controlled study. Clin Exp Rheumatol. 2001;19(3):265-70.

45. van der Weegen W, Wullems JA, Bos E, Noten H, van Drumpt RAM. No difference between intra-articular injection of hyaluronic acid and placebo for mild to moderate knee osteoarthritis: a randomized, controlled, double-blind trial. J Arthroplast. 2015;30(5):754-7.

46. Wobig M, Dickhut A, Maier R, Vetter G. Viscosupplementation with Hylan G-F 20: a 26-week controlled trial of efficacy and safety in the osteoarthritic knee. Clin Ther. 1998;20(3):410-23.

47. Bannuru RR, Natov NS, Dasi UR, Schmid CH, McAlindon TE. Therapeutic trajectory following intra-articular hyaluronic acid injection in knee osteoarthritis meta-analysis. Osteoarthr Cartil.

48. Trojian TH, Concoff AL, Joy SM, Hatzenbuehler JR, Saulsberry WJ, Coleman CIAMSSM. Scientific statement concerning Viscosupplementation injections for knee osteoarthritis. Clin J Sport Med. 2016;26(1):1-11.

49. Zoboli AAC, de Rezende MU, de Campos GC, Pasqualin T, Frucchi R, de Camargo OP. Prospective randomized clinical trial: single and weekly viscosupplementation. Acta Ortop Bras. 2013;21(5):271-5.

50. Greenwald RA. Oxygen radicals, inflammation, and arthritis: pathophysiological considerations and implications for treatment. Semin Arthritis Rheum. 1991; 20(4):219-40.

51. Bauer C, Niculescu-Morzsa E, Jeyakumar V, Kern D, Späth S, Nehrer S. Chondroprotective effect of high-molecular-weight hyaluronic acid on osteoarthritic chondrocytes in a co-cultivation inflammation model with M1 macrophages. J Inflamm. 2016;13(1)

52. Fioravanti A, Cantarini L, Chellini F, et al. Effect of hyaluronic acid (MW 500$730 \mathrm{kDa}$ ) on proteoglycan and nitric oxide production in human osteoarthritic chondrocyte cultures exposed to hydrostatic pressure. Osteoarthr Cartil. 2005; 13(8):688-96.

53. Gómez-Aristizábal A, Kim K, Viswanathan SA. Systematic study of the effect of different molecular weights of hyaluronic acid on mesenchymal stromal cell-mediated immunomodulation. PLoS One. 2016;1 1(1):e0147868.

54. Block J, Miller L. US-approved intra-articular hyaluronic acid injections are safe and effective in patients with knee osteoarthritis: systematic review and meta-analysis of randomized, saline-controlled trials. Clinical Medicine Insights: Arthritis and Musculoskeletal Disorders. 2013;57 https://doi.org/10.4137/ cmamd.s12743.

55. Finnoff JT, Hall MM, Adams E, et al. American medical Society for Sports Medicine (AMSSM) position statement: interventional musculoskeletal ultrasound in sports medicine. PM R. 2015;7(2):151-68. e112

56. Pham T, van der Heijde D, Altman RD, et al. OMERACT-OARSI initiative: osteoarthritis research society international set of responder criteria for osteoarthritis clinical trials revisited. Osteoarthritis and cartilage / OARS, Osteoarthritis Research Society. 2004;12(5):389-99.

\section{Submit your next manuscript to BioMed Central and we will help you at every step:}

- We accept pre-submission inquiries

- Our selector tool helps you to find the most relevant journal

- We provide round the clock customer support

- Convenient online submission

- Thorough peer review

- Inclusion in PubMed and all major indexing services

- Maximum visibility for your research

Submit your manuscript at www.biomedcentral.com/submit 